\title{
The range dependence of fluorescence anisotropy in molecular energy transfer
}

\author{
David L. Andrews and Gediminas Juzeliūnas ${ }^{\text {a) }}$ \\ School of Chemical Sciences, University of East Anglia, Norwich NR4 7TJ, United Kingdom
}

(Received 23 May 1991; accepted 2 July 1991)

\begin{abstract}
In the theory of excitation energy transfer it is generally considered that species initially excited by photoabsorption transfer their energy to other molecules by two distinct mechanisms, known as radiative and radiationless energy transfer. Recently it has been shown that the two mechanisms for energy transfer are in fact indistinguishable, each being the asymptotic limit of a unified mechanism involving virtual photon coupling. The familiar $R^{-6}$ dependence associated with Förster radiationless transfer is the short-range limit, while over longer distances retardation effects modify the radial dependence to $R^{-2}$, and the result is the classical radiative transfer law. For radiationless energy transfer, wide use is made of Galanin's result concerning fluorescence depolarization losses due to single-step transfer. Here Galanin's work is extended to obtain a general formula for the residual fluorescence anisotropy following energy transfer over arbitrary intermolecular distances. Hencc a conncction is established with the depolarization associated with reabsorption. In particular, it is shown that the anisotropy increases significantly from its initially low value over distances considerably less than those normally associated with radiative energy transfer. The necessary criteria for experimental identification of the transition from radiationless to radiative character are described, and model systems for their realization are considered.
\end{abstract}

\section{INTRODUCTION}

The migration of electronic excitation energy in condensed media has afforded a highly active field of both theoretical and experimental research for many years. ${ }^{1-3}$ Energy transfer plays an important role in a wide variety of physical, chemical, and biological processes such as intrinsic fluorescence quenching in enzymes and light energy harvesting in the photosynthetic unit. ${ }^{4,5}$ According to conventional wisdom (see, e.g., Ref. 6), the species initially excited by photoabsorption can transfer their energy to other sites by two distinct mechanisms, known as radiative and radiationless energy transfer. The former mechanism involves the emission and reabsorption of transverse photons, and is manifest over transfer distances $R$ which appreciably exceed the reduced photon wavelength $\lambda=\lambda / 2 \pi$. It is generally understood that the latter radiationless mechanism is mediated by a longitudinal (Coulombic) interaction, not involving transverse photons, which takes place over comparatively shortrange distances $R \ll \lambda$. So, while both of these resonant coupling mechanisms require an overlap between the appropriate emission and absorption spectra, their differences are commonly emphasised on the basis of other mechanistic features such as their apparently very different range dependences.

Recently it has been demonstrated that the radiative and radiationless mechanisms for energy transfer are, in fact, indistinguishable. ${ }^{7}$ Each is the asymptotic limit of a unified coupling mechanism involving virtual photon coupling, as has been proven using well-established methods of

\footnotetext{
" Permanent address: Institute of Theoretical Physics and Astronomy, $\mathrm{K}$. Poželos 54, 232600 Vilnius, Lithuania.
}

quantum electrodynamics. Thus the familiar $R^{-6}$ dependence associated with the Förster theory of dipole-dipole radiationless transfer ${ }^{8}$ appears as the short-range limit, whilst over longer distances retardation effects modify the form of the radial dependence to $R^{-2}$, and the result can be identified as the classical inverse-square radiative energy transfer law.

One of the most salient features of energy transfer concerns the fluorescence depolarization associated with the migration of excitation. Techniques based on measuring fluorescence depolarization (or decay of induced absorption anisotropy) prove to be extremely useful for obtaining information on both the energy transfer process ${ }^{9-11}$ and the internal structure of molecular systems. ${ }^{12-15}$ In the study of radiationless energy transfer, wide use is made of Galanin's result ${ }^{1,16}$ concerning polarization losses due to single-step transfer. In this paper, Galanin's work is extended to afford application to arbitrary transfer distances, thus establishing a connection with the depolarization associated with the radiative energy transfer. To our knowledge it is the first time that the effects of retardation have been considered in this connection; a discussion of the influence of such effects on the exciton spectra of molecular crystals is presented in the classic text by Craig and Walmsley. ${ }^{17}$

The paper is organized as follows. In the next section the unified theory, which has been shown to establish the connection between radiationless and radiative energy trans$\mathrm{fer}^{7}{ }^{7}$ is extended to incorporate the effects of molecular vibrational structure. Using these results the transfer-induced fluorescence depolarization is studied in Sec. III. Finally, in Sec. IV some concluding remarks are presented and possible applications are discussed. 


\section{DERIVATION OF A GENERALIZED ENERGY TRANSFER RATE}

In the Power-Zienau-Woolley formulation of molecular quantum electrodynamics ${ }^{18-20}$ the Coulombic interaction between molecules is represented by the propagation of transverse virtual photons, and it is the coupling between molecules and the radiation field which is responsible not only for molecular photoabsorption and spontaneous emission, but also for intermolecular energy transfer. In this formalism the Hamiltonian for the system can be written as

$$
H=H_{\mathrm{o}}+H_{\mathrm{int}}=H_{\mathrm{rad}}+H_{\mathrm{mol}}+H_{\mathrm{int}} \text {, }
$$

where $H_{\mathrm{rad}}$ is the radiation Hamiltonian, and

$$
\begin{aligned}
& H_{\mathrm{mol}}=\sum_{\xi} H_{\mathrm{mol}}(\xi), \\
& H_{\mathrm{int}}=\sum_{\xi} H_{\mathrm{int}}(\xi) .
\end{aligned}
$$

Here the sums are taken over all molecules in the system, $H_{\text {mol }}(\xi)$ is the normal Schrödinger operator for molecule $\xi$, and $H_{\text {int }}(\xi)$ represents the interaction between molecule $\xi$ and the radiation field. For the purposes of this paper it is sufficient to express the interaction Hamiltonian in the electric dipole approximation, as

$$
H_{\text {int }}(\xi)=-\epsilon_{0}^{-1} \mu(\xi) \cdot \mathbf{d}^{1}\left(\mathbf{R}_{\xi}\right) .
$$

where $\boldsymbol{\mu}(\xi)$ is the electric dipole operator and $\mathbf{R}_{\xi}$ the position vector of molecule $\xi$. In Eq. (4), $\mathbf{d}_{1}(\mathbf{r})$ is the transverse electric displacement field operator, which together with the magnetic induction field operator $\mathbf{b}(\mathbf{r})$ represent the dynamical variables of the electromagnetic field. In terms of these operators $H_{\text {rad }}$ is expressed as

$$
H_{\mathrm{rad}}=\frac{1}{2} \int\left\{\epsilon_{0}^{-1} \mathbf{d}^{12}+\epsilon_{0} c^{2} \mathbf{b}^{2}\right\} d^{3} \mathbf{r}
$$

The radiation Hamiltonian and the electric displacement operator may each be cast in terms of a mode expansion as follows:

$$
\begin{aligned}
H_{\mathrm{rad}}= & \sum_{\mathbf{k}, \lambda}\left[a^{+(\lambda)}(\mathbf{k}) a^{(\lambda)}(\mathbf{k})+\frac{1}{2}\right] \hbar c k, \\
\mathbf{d}^{\perp}(\mathbf{r})= & \sum_{\mathbf{k}, \lambda}\left(\frac{\hbar c k \epsilon_{0}}{2 V}\right)^{1 / 2} i\left\{\mathbf{e}^{(\lambda)}(\mathbf{k}) a^{(\lambda)}(\mathbf{k}) e^{i \mathbf{k} \cdot \mathbf{r}}\right. \\
& \left.-\overline{\mathbf{e}}^{(\lambda)}(\mathbf{k}) a^{+(\lambda)}(\mathbf{k}) e^{-i \mathbf{k} \cdot \mathbf{r}}\right\} .
\end{aligned}
$$

In both of the above expressions a sum is taken over all radiation modes characterized by wave vector $k$ and polarization vector $\mathrm{e}^{(\lambda)}(\mathbf{k}) ; a^{+(\lambda)}(\mathbf{k})$ and $a^{(\lambda)}(\mathbf{k})$ are the corresponding photon creation and annihilation operators and $V$ is the quantization volume.

In order to derive an expression for the rate $W_{f i}$ of molecular energy transfer associated with an initial system state $|i\rangle$ and a final state $|f\rangle$, we employ the normal methods of time-dependent perturbation theory with $H_{\text {int }}$ acting as the perturbation on basis states which are eigenstates of $H_{0}$. Results are then obtained from application of the generalized Fermi rule ${ }^{21}$

$$
W_{f i}=\frac{2 \pi}{\hbar}|\langle f|\widehat{T}| i\rangle|^{2} \delta\left(E_{f}-E_{i}\right),
$$

where $\widehat{T}$ is the transition operator given by

$$
\widehat{T}=\hat{T}^{(1)}+\hat{T}^{(2)}+\cdots
$$

with

$$
\begin{aligned}
& \widehat{T}^{(1)}=H_{\mathrm{int}}, \\
& \widehat{T}^{(2)}=H_{\mathrm{int}} \frac{1}{E_{i}-H_{0}+i s} H_{\mathrm{int}}, \quad s \rightarrow+0
\end{aligned}
$$

and higher order terms can be neglected for our purposes. The principal term $\widehat{T}^{(1)}$ can be regarded as representing both photoemission and photoabsorption by individual molecules, as shown by the time-ordered diagrams in Figs. 1(a) and $1(\mathrm{~b})$. The term $\widehat{T}^{(2)}$ features in the representation of molecular energy transfer, which is the subject of our present theory. Here we employ the terms donor and acceptor to indicate molecules between which energy is transferred. Elsewhere these are also referred to as fluorophore and quencher. Our theory accommodates situations in which donor and acceptor are either the same or chemically different species. Using the Condon principle to separate electronic and vibrational parts of the state vector, the initial and final states of the process and their energies are

$$
\begin{aligned}
& |i\rangle=|0\rangle\left|\Psi_{D^{*}} \Psi_{A}\right\rangle\left|\phi_{D^{*}}^{(n)}\right\rangle\left|\phi_{A}^{(m)}\right\rangle, \\
& |f\rangle=|0\rangle\left|\Psi_{D^{\prime}} \Psi_{A^{*}}\right\rangle\left|\phi_{D}^{(l)}\right\rangle\left|\phi_{A^{*}}^{(p)}\right\rangle, \\
& E_{i}=E_{D^{*}}^{(n)}+E_{A}^{(m)}, \\
& E_{f}=E_{D}^{(l)}+E_{A^{*}}^{(p)},
\end{aligned}
$$

the states of all other molecules in the system being unspecified. Here $|0\rangle$ denotes the photon vacuum state vector, $\left|\Psi_{D^{*}} \Psi_{A}\right\rangle$ and $\left|\Psi_{D} \Psi_{A^{*}}\right\rangle$ are the electronic parts of the initial and final state vectors. The indices $l(m)$ and $n(p)$ indicate vibrational levels of the donor (acceptor) in its ground and excited electronic states, respectively, with $E_{D}^{(l)}, E_{A}^{(m)}, E_{D^{*}}^{(n)}$

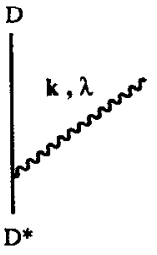

(a)

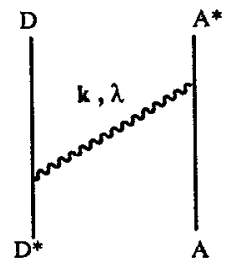

(c)

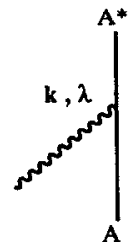

(b)

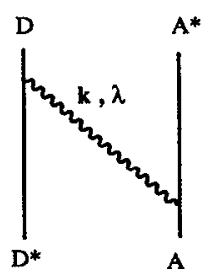

(d)
FIG. 1. Time-ordered diagrams for emission (a), absorption (b), and resonant energy transfer mediated by virtual photon exchange [(c) and (d)]. 
and $E_{A^{*}}^{(p)}$ being the corresponding electronic plus vibrational energies of donor and acceptor.

As depicted in Figs. 1(c) and 1(d), the quantum electrodynamical representation of the energy transfer arising from second order perturbation entails virtual photon coupling. The term "virtual" here relates to the fact that such photons are not observed. Their role is similar to that of the virtual molecular states which feature in scattering theory. The result for the matrix elements of the transition operator for molecular energy transfer calculated on the basis of the full quantum electrodynamical treatment is

$$
\left\langle f\left|\hat{T}^{(2)}\right| i\right\rangle=T_{A D}^{(2)}\left\langle\phi_{D}^{(l)} \mid \phi_{D^{*}}^{(n)}\right\rangle\left\langle\phi_{A^{*}}^{(p)} \mid \phi_{A}^{(m)}\right\rangle,
$$

where

$T_{A D}^{(2)}=\frac{K^{3} \mu_{A} \mu_{D} e^{i K R}}{4 \pi \epsilon_{0}}\left[\eta_{3}\left(\frac{1}{K^{3} R^{3}}+\frac{i}{K^{2} R^{2}}\right)-\eta_{1} \frac{1}{K R}\right]$

and

$$
\eta_{j}=\mathbf{e}_{A} \cdot \mathbf{e}_{D}-j\left(\mathbf{e}_{A} \cdot \mathbf{e}_{R}\right)\left(\mathbf{e}_{D} \cdot \mathbf{e}_{R}\right), \quad(j=1,3) .
$$

Here $T_{A D}^{(2)}$ is the electronic matrix element of the transition operator, $\eta_{1}$ and $\eta_{3}$ are the orientational factors, $\mu_{D}$ and $\mu_{A}$ are the absolute values of the transition dipole moments of donor and acceptor, and $\mathbf{e}_{D}, \mathbf{e}_{A}, \mathbf{e}_{R}$ are unit vectors oriented along the transition dipoles of the donor and acceptor molecules and their separation vector $\mathbf{R}$, respectively. In passing we note that $T_{A D}^{(2)}$ is related to the retarded dipole-dipole interaction tensor ${ }^{7,22} \theta_{i j}=\sigma_{i j}+i \tau_{i j}$ by $T_{A D}^{(2)}=\mu_{D_{i}} \mu_{A_{j}} \theta_{i j}$. Finally, in Eq. (17) the parameter $K$ corresponds to the transfer energy

$$
\hbar c K=\hbar \omega=\Delta E_{D}=\Delta E_{A}
$$

with

$$
\begin{aligned}
& \Delta E_{D}=E_{D^{*}}^{(n)}-E_{D}^{(l)}, \\
& \Delta E_{A}=E_{A^{*}}^{(p)}-E_{A}^{(m)} .
\end{aligned}
$$

Equations (16)-(18) properly accommodate the retardation effects which feature in both classical relativistic and quantum electrodynamical treatments of dipole-dipole coupling. Their significance with regard to polarization effects in intermolecular energy transfer will become apparent in the next section.

The excitation transfer rate as calculated from the generalised Fermi rule is now as follows:

$$
\begin{aligned}
W= & \frac{2 \pi}{\hbar} \sum_{n, l, m, p}\left|T_{A D}^{(2)}\right|^{2} \rho_{D^{*}}^{(n)} \rho_{A}^{(m)}\left|\left\langle\phi_{D}^{(l)} \mid \phi_{D^{*}}^{(n)}\right\rangle\right|^{2} \\
& \times\left|\left\langle\phi_{A^{*}}^{(p)} \mid \phi_{A}^{(m)}\right\rangle\right|^{2} \mid \delta\left(\Delta E_{D}-\Delta E_{A}\right) .
\end{aligned}
$$

Here the appropriate averaging over initial and summing over final states is carried out, $\rho_{D^{*}}^{(n)}$ and $\rho_{A}^{(m)}$ being the population distribution functions of the initial vibrational states of donor and acceptor. As in the Förster theory, the excitation transfer rate can be presented in terms of an overlap integral between donor and acceptor spectra

$$
W=\frac{2 \pi}{\hbar^{2}} \int I_{D^{*}}(\omega) I_{A}(\omega)\left|T_{A D}^{(2)}\right|^{2} d \omega
$$

with

$$
\begin{aligned}
& I_{D^{*}}(\omega)=\hbar \sum_{n, l} \rho_{D^{*}}^{(n)}\left|\left\langle\phi_{D}^{(l)} \mid \phi_{D^{*}}^{(n)}\right\rangle\right|^{2} \delta\left(\Delta E_{D}-\hbar \omega\right), \\
& I_{A}(\omega)=\hbar \sum_{m, p} \rho_{A}^{(m)}\left|\left\langle\phi_{A^{*}}^{(p)} \mid \phi_{A}^{(m)}\right\rangle\right|^{2} \delta\left(\Delta E_{A}-\hbar \omega\right),
\end{aligned}
$$

where $I_{D^{*}}(\omega)$ and $I_{A}(\omega)$ are proportional to the donor emission and acceptor absorption spectra, respectively, normalized as

$$
\int I_{D^{*}}(\omega) d \omega=\int I_{A}(\omega) d \omega=1 .
$$

Equation (23) can be rewritten in a form closer to the standard Förster formula as follows:

$$
W=\frac{9}{8 \pi \tau_{D} N_{A}} \int F_{D}(\omega) \alpha_{A}(\omega) K^{2} g(K R) d \omega
$$

with

$$
\begin{aligned}
g(K R)= & \eta_{3}^{2} \frac{1}{K^{6} R^{6}}+\left(\eta_{3}^{2}-2 \eta_{1} \eta_{3}\right) \frac{1}{K^{4} R^{4}} \\
& +\eta_{1}^{2} \frac{1}{K^{2} R^{2}} \\
F_{D}(\omega)= & \frac{\omega^{3} \tau_{D} \mu_{D}^{2}}{3 \epsilon_{0} \pi \hbar c^{3}} I_{D^{*}}(\omega), \\
\alpha_{A}(\omega)= & \frac{\pi \omega \mu_{A}^{2}}{3 \epsilon_{0} \hbar c} N_{A} I_{A}(\omega),
\end{aligned}
$$

and $K=\omega / c$. Here $F_{D}(\omega)$ is the donor radiation spectrum normalized to unity, $\alpha_{A}(\omega)$ is the Beer's law absorbance of the acceptor, $\tau_{D}$ is the radiative lifetime of the donor and $N_{A}$ is the number of acceptors per unit volume.

Equation (27) accommodates both radiative and radiationless energy transfer. At small donor-acceptor distances, $K R \ll 1$, it reduces to the usual rate for Förster-type nonradiative energy transfer, $W \sim \eta_{3}^{2} / R^{6}, \eta_{3}^{2}$ being the conventional orientational factor. For large distances, $K R \gg 1$, Eq. (27) gives the standard radiative result, $W \sim \eta_{1}^{2} / R^{2}$. The limiting cases thus differ in their orientational factors as well as their distance dependence. The orientational difference leads to completcly different transfer-induced fluorescence depolarization. In the next section the theory of fluorescence depolarization valid for arbitrary donor-acceptor separation is developed in detail. A fuller study of the energy transfer process per se will be presented elsewhere. ${ }^{23}$

\section{TRANSFER-INDUCED FLUORESCENCE DEPOLARIZATION}

For the usual nonradiative dipole-dipole mechanism for intermolecular energy transfer, the transfer rate on average depends only weakly on the mutual orientation of donors and acceptors [see Eq. (32) below for the average of the appropriate orientational factor, $\left.\eta_{3}^{2}\right]$. This is the reason for the well-known and considerable $(1 / 25)$ reduction of fluorescence anisotropy following a single act of energy transfer. ${ }^{1,16}$ By contrast in the radiative mechanism, energy transfer between species with parallel transition dipoles is greatly preferred [compare Eq. (31), in which the angle-dependent term is weighted by a factor of 7]. Consequently, the residual anisotropy after a single act of photon reabsorption is sub- 
stantially (seven times) greater than in the case of nonradiative transfer.

In the following a general formula is derived which connects and accommodates the above limiting cases. Here and throughout the paper rotational depolarization is assumed to be negligible. In order to arrive at a formula which demonstrates the effect of the relative orientation of donor and acceptor species in an ensemble, it is necessary to average Eq. (27) over the orientation of the vector $\mathbf{e}_{R}$ with respect to a donor-acceptor pair in fixed mutual orientation. We obtain the following results for the rotational averages of the orientational factors in Eq. (28):

$$
\overline{\eta_{1}^{2}}=\frac{1}{15}\left(7 \cos ^{2} \theta+1\right) \text {, }
$$

$$
\begin{aligned}
& \overline{\eta_{3}^{2}}=\frac{1}{3}\left(\cos ^{2} \theta+3\right), \\
& \overline{\eta_{1} \eta_{3}}=\frac{1}{3} \overline{\eta_{3}^{2}},
\end{aligned}
$$

where $\cos \theta=\mathbf{e}_{A} \cdot \mathbf{e}_{D}$. Substituting these results into Eqs. (27)-(30) we obtain

$$
\overline{W(R)} \propto\left(3 y_{6}+y_{4}+7 y_{2}\right) \cos ^{2} \theta+\left(9 y_{6}+3 y_{4}+y_{2}\right)
$$

with

$y_{n}=\frac{1}{R^{n}} \int K^{2-n} F_{D}(\omega) \alpha_{A}(\omega) d \omega \quad(n=2,4,6)$.

Therefore, the properly normalized orientational distribution function for excited acceptors, $f(\theta, R)$, is given by

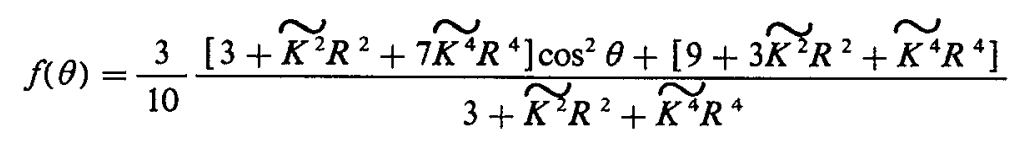

with

$$
\widetilde{K^{n}}=\frac{\int K^{n} F_{D}(\omega) \alpha_{A}(\omega) \omega^{-4} d \omega}{\int F_{D}(\omega) \alpha_{A}(\omega) \omega^{-4} d \omega}
$$

Of special interest is the fluorescence anisotropy defined by

$$
r=\frac{I_{\|}-I_{\perp}}{I_{\|}+2 I_{\perp}}
$$

Here $I_{\|}$and $I_{1}$ are the components of the fiuorescence intensity polarized parallel and perpendicular to the polarization of the excitation light, respectively. In the case where fluorescence occurs directly from the molecule which absorbs the incident light (the donor), the anisotropy is designated $r_{0}$; where fluorescence occurs following single-step intermolecular energy transfer to another molecule (the acceptor) the anisotropy is designated $r_{1}$. The value of $r_{0}$, if donor intramolecular relaxation produces no change of electronic state, has its theoretical maximum of 0.4.

It is the result for $r_{1}$ which is of principal interest; the fluorescence anisotropy following a chain of energy transfer events can be directly calculated from this result. In terms of $r_{0}$, the acceptor anisotropy $r_{1}$ can be expressed as ${ }^{24}$

$$
r_{1}=\left\langle P_{2}(\cos \theta)\right\rangle r_{0}
$$

where $P_{2}(\cos \theta)=\left(3 \cos ^{2} \theta-1\right) / 2$ is the second-order Legendre polynomial and the angular brackets denote the distributional average

$$
\left\langle P_{2}(\cos \theta)\right\rangle=\frac{1}{2} \int_{0}^{\pi} P_{2}(\cos \theta) f(\theta) \sin \theta d \theta .
$$

Substituting expression (36) for $f(\theta)$ into Eq. (40) we obtain the final and most general result

$$
r_{1}(R)=\frac{r_{0}}{25}\left(\frac{\widetilde{7 K^{4}} R^{4}+\widetilde{K^{2}} R^{2}+3}{\widetilde{K^{4} R^{4}+\widetilde{K}^{2} R^{2}+3}}\right) .
$$

The above equation is valid for arbitrary separations $R$. As shown in Eqs. (45) and (46) below, the familiar short- and long-range results are the asymptotes of this formula. It should be pointed out, that in general the residual anisotropy
$r_{1}(R)$ depends not only on the transfer distance $R$, but also on the shapes of the spectral lines [through the averages $K^{2}$ and $\widehat{K}^{4}$ appearing in expression (41)]. However, as the widths of the absorption and emission lines are considerably smaller than the photon frequency, Eq. (41) can be rewritten without any significant loss of generality as

$$
r_{1}(R)=\frac{r_{0}}{25}\left(\frac{7(\widetilde{K} R)^{4}+(\widetilde{K} R)^{2}+3}{(\widetilde{K} R)^{4}+(\widetilde{K} R)^{2}+3}\right),
$$

where $\widetilde{K}$ is the average value of $K$ calculated by use of Eq. (37) with $n=1$. In the case where the absorption and cmission lines are of Gaussian shape $\left\{F_{D} \sim \omega^{3}\right.$ $\exp \left[-\left(\omega-\omega_{D}\right)^{2} / 2 \sigma^{2}\right], \quad \alpha_{A} \sim \omega \exp \left[-\left(\omega-\omega_{A}\right)^{2} /\right.$ $\left.\left.2 \sigma^{2}\right]\right\}$, we have

$$
\begin{aligned}
& \widetilde{K^{2}}=\widetilde{K^{2}}\left[1+\frac{1}{2}\left(\frac{\sigma}{\widetilde{\omega}}\right)^{2}\right], \\
& \widetilde{K^{4}}=\widetilde{K^{4}}\left[1+3\left(\frac{\sigma}{\tilde{\omega}}\right)^{2}+\frac{3}{4}\left(\frac{\sigma}{\tilde{\omega}}\right)^{4}\right] .
\end{aligned}
$$

This means that if, for example, the ratio $\sigma / \widetilde{\omega}$ is 0.1 then the error made by using expression (42) instead of Eq. (41) is less than a few percent. Moreover both formulas (41) and (42) give the correct asymptotes at small and large distances:

$\widetilde{K} R<1$. Here Eqs. (41) and (42) reduce to the usual Galanin result

$$
r_{1}=r_{1}^{\text {non-rad }}=\frac{1}{25} r_{0} .
$$

$\widetilde{K} R>1$. Here we obtain the result for the depolarization associated with photon recapture

$$
r_{1}=r_{1}^{\mathrm{rad}}=\frac{7}{25} r_{0} \text {. }
$$

The distance dependence of the relative residual anisotropy $r_{1}(R) / r_{0}$ calculated according to Eq. (42) is presented in Fig. 2. 


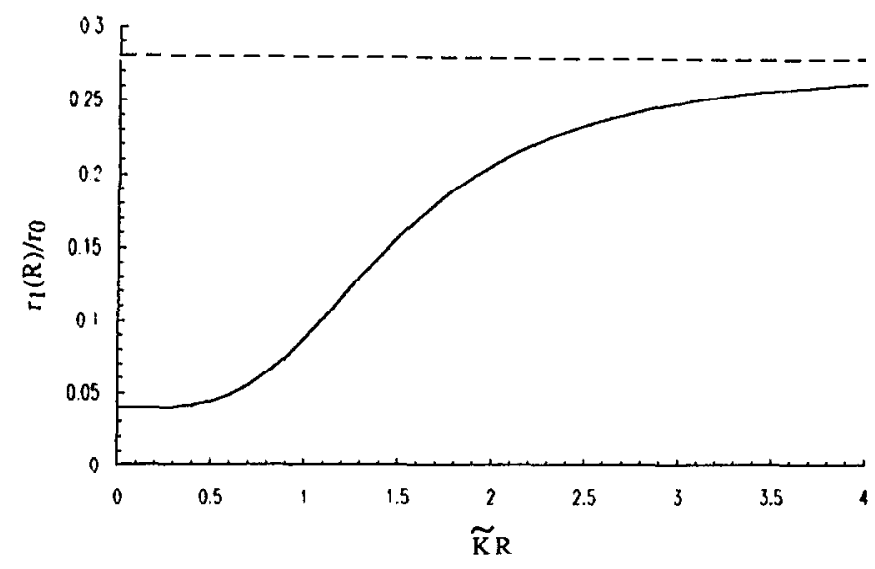

FIG. 2. Relative anisotropy, $r_{1}(R) / r_{0}$, plotted as a function of $\widetilde{K} R$ according to Eq. (42). The dotted line represents the limiting value associated with radiative transfer.

\section{DISCUSSION}

In this paper we have extended Galanin's result for radiationless energy transfer to be applicable to arbitrary transfer distances $R$, thus cstablishing a connection with the depolarization associated with photon reabsorption. The results obtained show that the extent of anisotropy in the distribution of excited acceptors, as measured by the fluorescence anisotropy $r_{1}$, has a very marked dependence on $R$. For $R<\lambda(\lambda=\lambda / 2 \pi=1 / \widetilde{K})$ our general formulas $(41)$ and (42) reduce to Galanin's result that a single act of energy transfer produces a 25-fold decrease in the anisotropy. This justifies the usual neglect of polarization contributions associated with molecules other than the initially excited species. ${ }^{9-11}$ In contrast, in the case of photon reabsorption taking place at distances $R \gg \lambda$ the residual anisotropy $r_{1}$ is considerably higher and cannot usually be ignored.

What is perhaps most noteworthy, however, is the fact that $r_{1}$ changes to a significant value at distances much less than those normally associated with radiative energy transfer (see Fig. 2). For example, with a donor-acceptor separation $R=1.5 / \widetilde{K}=0.75 \lambda / \pi$, the relative anisotropy $r_{1} / r_{0}$ attains the value of $3 / 25$, considerably higher than the result for the radiationless transfer, as follows from Eq. (42).

Whilst the range dependence of the fluorescence depolarization is of intrinsic interest, manifesting retardation features which are not widely known to appear in such a connection, it is also of interest to speculate on potential systems in which the average transfer distance lies somewhere in the region of the reduced wavelengths corresponding to the energy being transferred $(\widetilde{K} R \sim 1)$. In this critical region neither the usual radiationless nor the radiative result is correct. To realize such a system in practice, the following criteria should be satisfied as fully as possible.

First, the ideal system should comprise microsystems in which each donor is surrounded by acceptors at a relatively fixed distance in the critical region. Second, the acceptors should not be excited by the radiation which initially excites donors. Thus the donor and acceptor species must normally be chemically different and their absorption spectra should not fully overlap. In the same way it is necessary for the donor and acceptor fluorescence spectra to be separable, so that the acceptor fluorescence can be registered in a wavelength region where there is no influence of the donor signal.

Third, the probability of donor-acceptor energy transfer should be sufficiently high to make the process measurable. This probability can be increased by having a group (domain) of donors centrally positioned within a spherical shell of acceptors densely packed together in several layers. Both the domain size and the shell thickness should, of course, be of a dimension considerable smaller than the shell radius. Moreover, it is necessary to minimize depolarization due to donor-donor or acceptor-acceptor transfer. Hence donor molecules forming a domain should have a high degree of orientational order, and the acceptor species must also be highly orientationally ordered over distances equal to the length of excitation diffusion between acceptor molecules of the shell, $l_{\text {diff }}$, usually not exceeding a few tens of nanometers. This is entirely realistic since the acceptor shell will typically need to be some hundreds of nanometers across.

Last, the probability of energy transfer from any one donor to an acceptor belonging to another sphere must be much less than the probability of transfer to an acceptor in its own sphere. Hence the concentration of the spherical microsystems must be kept low, and/or the optical pathlength be sufficiently short that a photon emitted by donor can normally be absorbed only by its own acceptor surroundings. Having such a system one can, by registering acceptor luminescence or its anisotropy, distinguish the transition from radiationless to radiative character in the donor-acceptor transfer. The polarization method offers considerable advantages over absolute measurements of the luminescence which would otherwise require knowledge of the number of donors per domain and the number of acceptors per sphere.

In conclusion, while the production of a system such as described above is technologically demanding, the principal result of this paper also has intrinsic interest from a purely scientific point of view, since it establishes the continuous connection between fluorescence features associated with radiationless and radiative molecular energy transfer.

\section{ACKNOWLEDGMENTS}

One of the authors (G. J.) wishes to thank the School of Chemical Sciences, University of East Anglia for their hospitality and the Royal Society for financial support during his stay in the UK.

${ }^{1}$ V. M. Agranovich and M. D. Galanin, Electronic Excitation Energy Transfer in Condensed Matter (North-Holland, Amsterdam, 1982).

${ }^{2}$ V. M. Kenkre and P. Reineker, Exciton Dynamics in Molecular Crystals and Aggregates (Springer, Berlin, 1982).

${ }^{3}$ Spectroscopy and Excitation Dynamics of Condensed Molecular Systems, edited by V.M. Agranovich and R. M. Hochst rasser (North-Holland, Amsterdam, 1983).

${ }^{4}$ The Photosynthetic Bacteria, edited by R. K. Clayton and W. R. Sistrom (Plenum, New York, 1978).

${ }^{5}$ Perspectives in Photosynthesis, edited by J. Jortner and B. Pullman (Kluwer Academic, Dordrecht, 1990).

oJ. E. Bernard, D. E. Berry, and F. Williams, in Energy Transfer Processes in Condensed Matter, edited by B. Di Bartolo (Plenum, New York, 1984), p. 1. 
'D. L. Andrews, Chem. Phys. 135, 195 (1989).

${ }^{8}$ T. Förster, Z. Naturforsch. Teil A 4, 321 (1949).

${ }^{9}$ C. R. Gochanour and M. D. Fayer, J. Phys. Chem. 85, 1989 (1981).

${ }^{10}$ P. A. Anfinrud and W. S. Struve, J. Phys. Chem. 91, 5058 (1987).

${ }^{11} \mathrm{~K}$. Sienicki, H. Itagaki, and W. L. Mattice, J. Chem. Phys. 91, 4515 (1989).

${ }^{12}$ V. Sundström, T. Gillbro, R. A. Gadonas, and A. Piskarskas, J. Chem. Phys. 89, 2754 (1988).

${ }^{13}$ Z. G. Fetisova, A. M. Freiberg, and K. E. Timpmann, Nature (London) 334, 633 (1988).

${ }^{14}$ M. Miller, R. P. Cox, and T. Gillbro, Biophys. Biochim. Acta 1057, 187 (1991).

${ }^{15}$ G. Juzeliūnas, Chem. Phys. 151, 169 (1991).
${ }^{16}$ M. D. Galanin, Trudy FIAN SSSR 5, 341 (1950).

${ }^{17}$ D. P. Craig and S. H. Walmsley, Excitons in Molecular Crystals. Theory and Applications (Benjamin, New York, 1968).

${ }^{18}$ E. A. Power and S. Zienau, Philos. Trans. R. Soc. London Ser. A 251, 427 (1959).

${ }^{19}$ R. G. Woolley, Proc. R. Soc. London Ser. A 321, 557 (1971).

${ }^{20}$ D. P. Craig and T. Thirunamachandran, Molecular Quantum Electrodynamics (Academic, London, 1984).

${ }^{21}$ L. S. Rodberg and R. M. Thaler, Introduction to the Quantum Theory of Scattering (Academic, New York, 1967), Chap. 8.

${ }^{22}$ D. L. Andrews and B. S. Sherborne, J. Chem. Phys. 86, 4011 (1987).

${ }^{23}$ D. L. Andrews and G. Juzeliūnas (to be published).

${ }^{24}$ R. E. Dale and J. Eisinger, Biopolymers 13, 1573 (1974). 